\title{
The Infra-Red Absorption Spectrum of Methyl Alcohol
}

\author{
Avis Borden and E. F. Barker \\ University of Michigan, Ann Arbor, Michigan
}

(Received June 20, 1938)

\begin{abstract}
Using a grating spectrometer having a $\mathrm{KBr}$ foreprism the spectrum of methyl alcohol vapor was studied in the region from 2.5 to $26 \mu$. This molecule has bands at 3683 , $2978,2845,2054,1477,1455,1340,1034.18 \mathrm{~cm}^{-1}$ and a very broad band extending from 860 to beyond $380 \mathrm{~cm}^{-1}$. These bands are typical perpendicular and parallel bands and indicate that the molecule is only slightly asymmetric.

From the fine structure of the $1034.18 \mathrm{~cm}^{-1}$ parallel band and from certain assumptions about the structure of the molecule the two largest moments of inertia, $A$ and $B$, were found to be 35.18 and $33.83 \times 10^{-40} \mathrm{~g} \mathrm{~cm}^{2}$, respectively. It was not possible to measure $C$ directly but it was estimated to be approximately $6.8 \times 10^{-40} \mathrm{~g} \mathrm{~cm}^{2}$.

The low frequency band is probably due to the vibration of the hydroxyl hydrogen atom perpendicular to the $\mathrm{O}-\mathrm{H}$
\end{abstract}

bond and perpendicular to the figure axis of the molecule. The presence of this band indicates that the hydroxyl group is not free to rotate, at least in the ground state. There is evidence of rotation in the structure of the band near the high frequency edge. The spacing increases toward high frequencies and finally there is a group of lines having a spacing of about $40 \mathrm{~cm}^{-1}$ between 600 and 860 $\mathrm{cm}^{-1}$. Since these lines are very weak and occur at such high frequencies it appears that free rotation exists only in states of high excitation. A quantitative study of free rotation in a symmetric molecule has been made. This investigation indicates that in a first approximation free rotation should give rise to groups of lines having a spacing of about $40 \mathrm{~cm}^{-1}$ between each group.

\section{INTRODUCTION}

A STUDY of the infra-red absorption spectrum of methyl alcohol has been made by Bartholomé and Sachsse ${ }^{1}$. using a prism spectrometer. Earlier studies have also been made by Titeica $^{2}$ and Lecomte. ${ }^{3}$ In addition, the Raman spectrum of methyl alcohol has been well established by the work of a number of investigators. ${ }^{4}$ These studies were of value in establishing the positions of the bands but they were unable to give information about their character. Moreover, due to the absorption of the rocksalt prisms used in the infra-red investigations it was not possible to work with wave-lengths longer than $16 \mu$.

In the present work the absorption spectrum was studied under high dispersion using the grating spectrometer designed by Hardy. ${ }^{5}$ With this instrument it was possible to resolve lines having a separation of less than $1 \mathrm{~cm}^{-1}$ over most

\footnotetext{
1 E. Bartholomé and H. Sachsse, Zeits. f. physik. Chemie B30, 40 (1935).

2 R. Titeica, Comptes rendus Acad. Sci., Paris 196, 391 (1933).

$3 \mathrm{~J}$. Lecomte, Comptes rendus Acad. Sci., Paris 180, 825 (1925).

${ }^{4}$ J. O. Halford, L. C. Anderson, G. H. Kissin, J. Chem. Phys. 5, 927 (1937). V. S. Mizushima, Y. Morina, G. Okamoto, Bull. Chem. Soc. Japan 11, 698 (1936). K. W. F. Kolhrausch, Der Smekal-Raman Effekt, p. 309.

5 J. D. Hardy, Phys. Rev. 38, 2162 (1931).
}

of the range. By using a $\mathrm{KBr}$ foreprism it was possible to extend the spectral region to $26 \mu$. This extension of the spectral region and the ability to resolve the fine structure of the bands made it possible to assign the frequencies more accurately and to gain further information about the structure of the molecule.

The form of the methyl alcohol molecule is known from sterochemical evidence and from infra-red data on other similar molecules. The carbon atom lies within a tetrahedron formed by hydrogen atoms at three of its corners and by the hydroxyl group at the fourth. The hydroxyl hydrogen atom stands out at an angle, somewhat greater than a right angle, from the $\mathrm{C}-\mathrm{O}$ line.

Such a molecule should possess twelve fundamental frequencies. Since the molecule is very nearly symmetrical, however, it turns out that only nine of the frequencies are distinct, three being double perpendicular frequencies. Of these nine, six correspond to the frequencies found for the methyl halides. Following Dennison's notation, ${ }^{6}$ the first six frequencies are assigned to those modes of vibration which also occur in the methyl halides. $\nu_{7}, \nu_{8}$ and $\nu_{9}$ are assigned to the frequencies associated with vibrations within the hydroxyl group.

\footnotetext{
${ }^{6}$ D. M. Dennison, Rev. Mod. Phys, 3, 280 (1931).
} 


\section{EXPERIMENTAL RESULTS}

The positions of the bands, the approximate amounts of gas required, the gratings used and the slit widths are given in Table I. A $25 \mathrm{~cm}$

TABLE I. Position of the bands.

\begin{tabular}{|c|c|c|c|c|}
\hline \multicolumn{2}{|c|}{$\begin{array}{l}\text { FREQUENCY } \\
\text { IN CM }^{-1}\end{array}$} & $\begin{array}{c}\text { PRESSURE } \\
\text { CM HG }\end{array}$ & $\begin{array}{l}\text { GRATING LINES } \\
\text { PER INCH }\end{array}$ & $\begin{array}{c}\text { Silt } \\
\text { WIDTH } \\
\text { IN } \\
\text { CM }^{-1}\end{array}$ \\
\hline $\begin{array}{r}\nu_{7} \\
\nu_{2} \\
\nu_{1} \\
2 \nu_{5} \\
\nu_{4} \\
\nu_{3} \\
\nu_{6} \\
\nu_{5} \\
\\
\nu_{8}\end{array}$ & $\begin{array}{l}3683 \\
2978 \\
2845 \\
2054 \\
1477 \\
1455 \\
1340 \\
1034.18 \\
860-650 \\
630-380\end{array}$ & $\begin{array}{l}5 \\
1 \\
1 \\
9 \\
9 \\
9 \\
9 \\
0.5\end{array}$ & $\begin{array}{l}4800 \\
4800 \\
4800 \\
4800 \\
1200 \\
1200 \\
1200 \\
2400 \\
1200 \\
\left\{\begin{array}{c}1200 \\
360 \text { (2nd order) }\end{array}\right.\end{array}$ & $\begin{array}{l}2.0 \\
1.3 \\
1.3 \\
0.65 \\
1.8 \\
1.8 \\
1.5 \\
0.5 \\
0: 8 \\
0.5 \\
0.5\end{array}$ \\
\hline
\end{tabular}

absorption cell with $\mathrm{KBr}$ windows was used for all the bands except for the region between 860 and $650 \mathrm{~cm}^{-1}$. Here it was necessary to use a meter cell to obtain sufficient absorption. It was impossible, however, to measure the pressure of the gas in the long cell since it could not be evacuated.

$\nu_{1}$ and $\nu_{2}$ are very intense bands which have their centers at 2845 and $2978 \mathrm{~cm}^{-1}$, respectively, Fig. 1. These two frequencies are associated with the vibrations of the three hydrogen atoms in the methyl group, $\nu_{1}$ being a parallel vibration and $\nu_{2}$ a double perpendicular vibration. Although the amplifier was used at its limit of sensitivity and the slits were made as narrow as possible it was impossible to resolve the fine structure of either of these bands.

$\nu_{3}$ and $\nu_{4}$ are also associated with vibrations within the methyl group. $\nu_{3}$ is a parallel band having its zero branch at $1455 \mathrm{~cm}^{-1} . \nu_{4}$ is another double perpendicular band which has its center at $1477 \mathrm{~cm}^{-1}$, Fig. 2. These two bands fall in a region where the atmospheric absorption due to water vapor is very intense. For this reason it was very difficult to resolve the fine structure of the bands except for a few lines near the center of the perpendicular band. These lines have the frequencies: $1463.2,1471.7,1479.9 \mathrm{~cm}^{-1}$. It was impossible to obtain the fine structure of the parallel band even with a finer grating and narrower slits.

These four methyl frequencies found in methyl alcohol are in good agreement with those found in the methyl halides and are almost identical with the frequencies of methyl fluoride. ${ }^{7}$ In the spectra of the methyl halides, however, another parallel band is usually observed between $\nu_{1}$ and $\nu_{2}$, which has been interpreted as the overtone of $\nu_{4},{ }^{8}$ This frequency was not observed in the spectrum of methyl alcohol although the region was examined carefully with different amounts of gas. The perpendicular band is not symmetrical, however, and it may be that there is a faint band on the low frequency side of it.

The double perpendicular band $\nu_{6}$ consists of the vibration between the methyl and hydroxyl

${ }^{7}$ W. H. Bennett and C. F. Meyer, Phys. Rev. 32, 888 (1928).

${ }^{8}$ A. Adel and E. F. Barker, J. Chem. Phys. 2, 627 (1934).

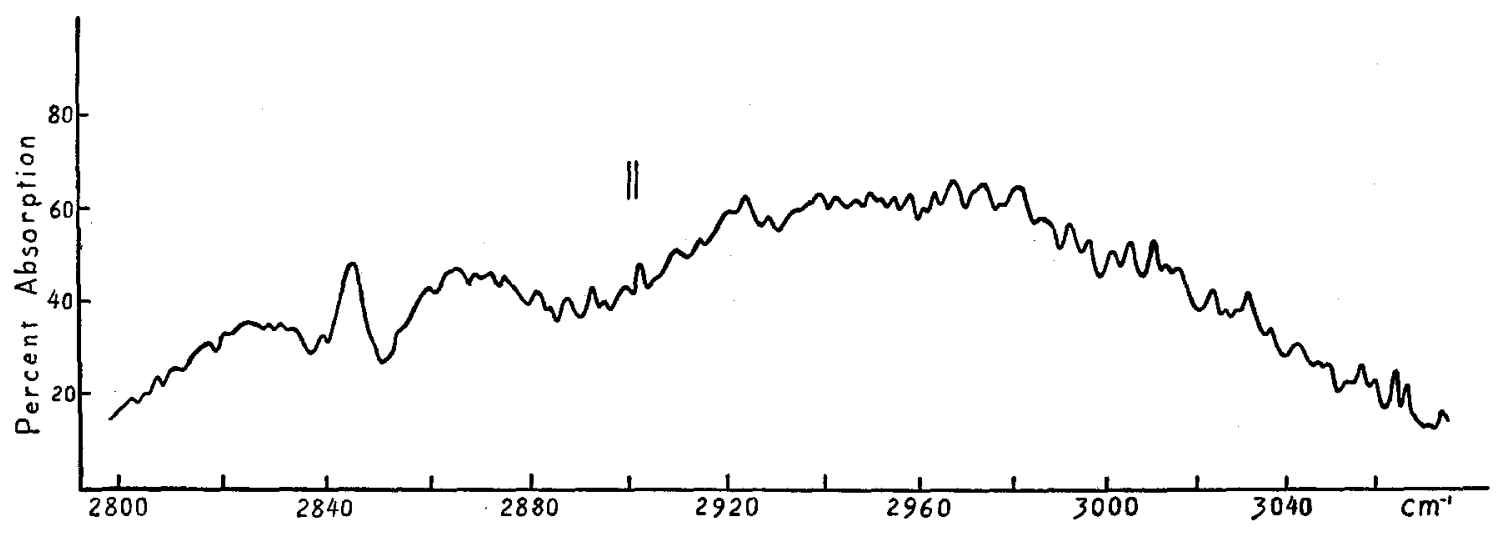

FIG. 1. The bands $\nu_{1}$ at $2845 \mathrm{~cm}^{-1}$ and $\nu_{2}$ at $2978 \mathrm{~cm}^{-1}$. 


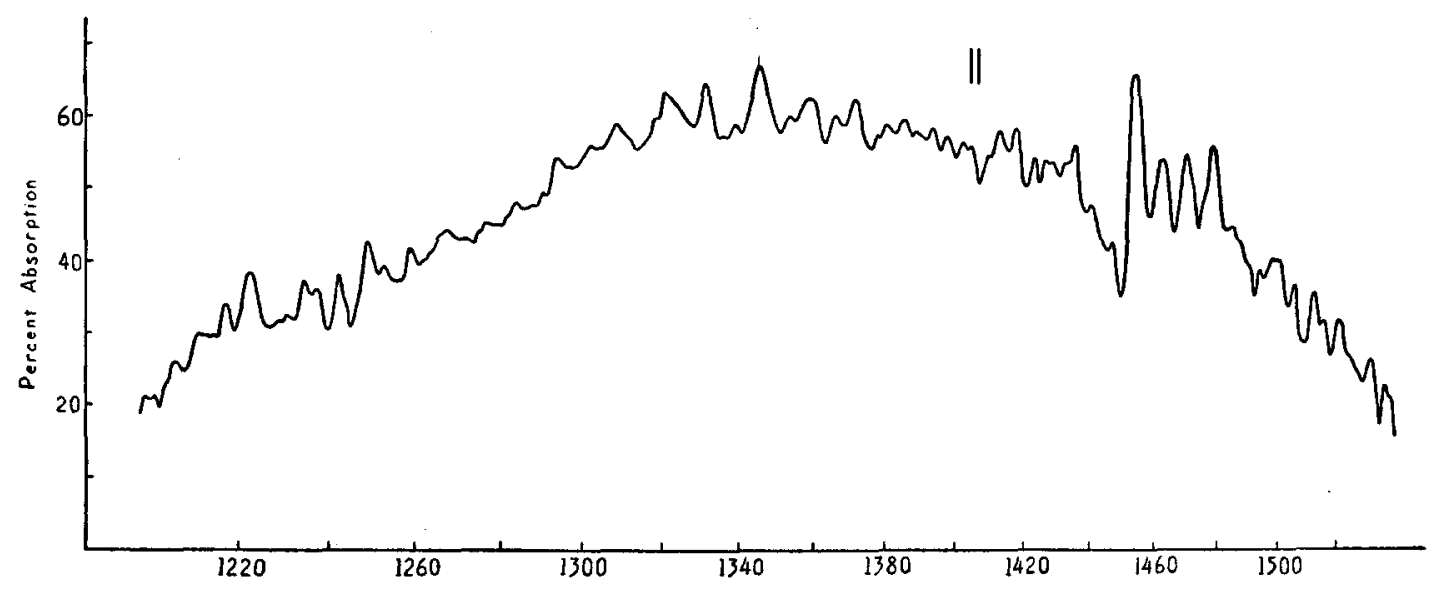

FIG. 2. The bands $\nu_{3}$ at $1455 \mathrm{~cm}^{-1}, \nu_{4}$ at $1477 \mathrm{~cm}^{-1}$ and $\nu_{6}$ at $1340 \mathrm{~cm}^{-1}$.

groups and lies at $1340 \mathrm{~cm}^{-1}$, Fig. 2. One might expect the two frequencies in this band to be separated, as was the case in the corresponding perpendicular band of formaldehyde, studied by Nielsen. ${ }^{9}$ Such a splitting is not immediately apparent from the structure of the band. There is, however, considerable atmospheric absorption out to $1350 \mathrm{~cm}^{-1}$ which introduces uncertainties in the positions and intensities of the fine structure lines. The band does appear to be considerably broader than most of the other perpendicular bands of this molecule, thus indicating the possibility of a double frequency. Although the background is fairly clear beyond $1350 \mathrm{~cm}^{-1}$ the band does not show much structure except near the center and far out on the low frequency edge. The central lines have the frequencies : $1321.3,1331.8,1348.4,1359.5 \mathrm{~cm}^{-1}$.

The parallel vibration $\nu_{5}$ between the methyl and hydroxyl groups gives rise to a very intense band at $1034.18 \mathrm{~cm}^{-1}$, Fig. 3 . The fine structure in this band is well resolved in both branches and fits the quadratic equation:

$$
\nu=1034.18+1.5975 m-0.0088 m^{2}
$$

where $m$ is the number of the line counting from the center. The calculated and observed frequencies are given in Table II. Most of the calculated values fit the observed frequencies within $0.05 \mathrm{~cm}^{-1}$.

The overtone of $\nu_{5}$ occurs as a weak parallel band at $2054 \mathrm{~cm}^{-1}$, Fig. 4. The fine structure

\footnotetext{
${ }^{9}$ H. H. Nielsen, J. Chem. Phys. 5, 818 (1937).
}

could be resolved only in the negative branch. Here the spacing agrees well with the spacing found in $\nu_{5}$.

$\nu_{7}$ consists of the motion of the hydroxyl hydrogen atom along the $\mathrm{O}-\mathrm{H}$ bond and lies at $3683 \mathrm{~cm}^{-1}$, Fig. 5. The atmospheric absorption due to water vapor is particularly strong here and it was impossible to obtain more than just the envelope of the band. The central line, however, occurs between two water vapor lines and was observed several times. This band has the TABLE II. Frequencies of the fine structure lines in the $1034.18 \mathrm{~cm}^{-1}$ parallel band.

\begin{tabular}{crr|rrr}
\hline \hline & & & & \\
$m$ & OBSERVED & $\begin{array}{c}\text { CALCU- } \\
\text { LATED }\end{array}$ & $m$ & OBSERVED & $\begin{array}{c}\text { CALCU- } \\
\text { LATED }\end{array}$ \\
\hline-27 & 983.78 & 983.82 & -2 & & 1031.94 \\
-26 & 986.68 & 986.69 & -1 & & 1032.57 \\
-25 & 988.72 & 988.74 & 0 & & 1034.18 \\
-24 & 990.78 & 990.78 & 1 & & 1035.77 \\
-23 & 992.76 & 992.78 & 2 & & 1037.34 \\
-22 & 994.69 & 994.78 & 3 & 1038.79 & 1038.89 \\
-21 & 996.77 & 996.76 & 4 & 1040.34 & 1040.43 \\
-20 & 998.71 & 998.71 & 5 & 1041.92 & 1041.94 \\
-19 & 1000.68 & 1000.65 & 6 & 1043.41 & 1043.45 \\
-18 & 1002.57 & 1002.57 & 7 & 1044.94 & 1044.93 \\
-17 & 1004.52 & 1004.48 & 8 & 1046.40 & 1046.40 \\
-16 & 1006.36 & 1006.37 & 9 & 1047.75 & 1047.85 \\
-15 & 1008.26 & 1008.24 & 10 & 1049.23 & 1049.28 \\
-14 & 1010.01 & 1010.09 & 11 & 1050.64 & 1050.69 \\
-13 & 1011.94 & 1011.92 & 12 & 1052.12 & 1052.08 \\
-12 & 1013.78 & 1013.74 & 13 & 1053.55 & 1053.46 \\
-11 & 1015.62 & 1015.55 & 14 & 1054.86 & 1054.93 \\
-10 & 1017.40 & $\mathbf{1 0 1 7 . 3 2}$ & 15 & 1056.18 & 1056.16 \\
-9 & 1019.16 & 1019.09 & 16 & 1057.55 & 1057.49 \\
-8 & 1020.86 & $\mathbf{1 0 2 0 . 8 4}$ & 17 & 1058.82 & 1058.80 \\
-7 & 1022.57 & 1022.57 & 18 & 1060.02 & 1060.09 \\
-6 & 1024.26 & $\mathbf{1 0 2 4 . 2 7}$ & 19 & 1061.23 & 1061.35 \\
-5 & 1025.94 & 1025.98 & 20 & 1062.50 & 1062.61 \\
-4 & 1027.58 & 1027.65 & 21 & 1063.80 & 1063.84 \\
-3 & 1029.36 & 1029.31 & & & \\
\hline & & & & & \\
\hline & & & & & \\
\hline
\end{tabular}




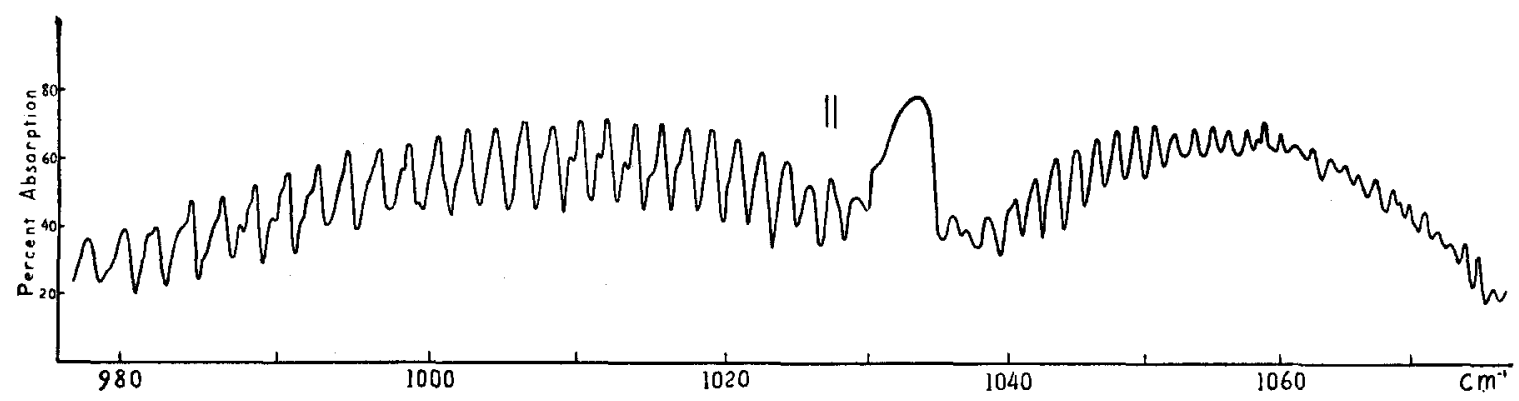

FIG. 3. The parallel bands $\nu_{5}$ at $1034.18 \mathrm{~cm}^{-1}$.

appearance of a perpendicular band with possibly a weak parallel component.

In the Raman spectrum of liquid methyl alcohol $\nu_{7}$ was found at $3400 \mathrm{~cm}^{-1}$ instead of at $3683 \mathrm{~cm}^{-1}$. This discrepancy is not an error in the results but is characteristic of the molecule when it passes from the liquid to the gaseous state. Errera and Mollet ${ }^{10}$ have plotted this band in the infra-red using liquid alcohol and found it at $3 \mu$. When they diluted the alcohol with $\mathrm{CCl}_{4}$ this band disappeared and a faint one was detected at $2.7 \mu$. They conclude that the $3 \mu$ band is due to association and that the fundamental is at $2.7 \mu$. The dilution of the alcohol breaks up the association of the molecules. This agrees well with the results of the present investigation since no band was found at $3 \mu$ using alcohol vapor.

Another hydroxyl frequency $\nu_{9}$ consists of the motion of the hydrogen atom perpendicular to the $\mathrm{O}-\mathrm{H}$ bond and nearly parallel to the figure axis of the molecule. This band was never found in the infra-red. The Raman spectrum gives this frequency at $1107 \mathrm{~cm}^{-1}$. In the molecule $\mathrm{CH}_{3} \mathrm{OD}$ this band also is affected by association." Its frequency is about $80 \mathrm{~cm}^{-1}$ lower than the Raman spectrum predicts. If a similar shift takes place in ordinary methyl alcohol this band should

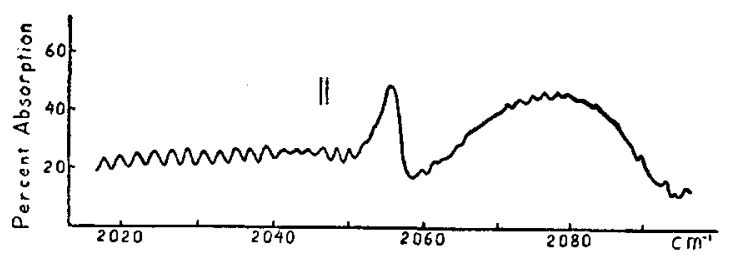

FIG. 4. The band $2 \nu_{5}$ at $2054 \mathrm{~cm}^{-1}$.

\footnotetext{
${ }^{10}$ J. Errera, Trans. Faraday Soc. London 33, 122 (1937). II $G$. Bosschieter and E. F. Barker, paper immediately following in J. Chem. Phys.
}

lie near $1030 \mathrm{~cm}^{-1}$ which is in the midst of the very intense $1034.18 \mathrm{~cm}^{-1}$ band, $\nu_{5}$. The 1034.18 $\mathrm{cm}^{-1}$ band does have irregularities in its fine structure which may be due to the presence of this other band. But the hydroxyl frequency is essentially parallel and one should expect to find a zero branch somewhere. Although the region was studied a number of times with different amounts of gas no zero branch could be found unless it coincides with the zero branch of $\nu_{5}$. These two frequencies are of the same symmetry class, however, and one would expect a resonance interaction to separate them. It is not possible to use much gas in this region before the absorption becomes complete. The hydroxyl frequency may be too weak to be detected here.

Finally, there is a frequency $\nu_{8}$ in which the hydrogen atom moves perpendicular to the $\mathrm{O}-\mathrm{H}$ bond and perpendicular to the axis of the molecule. If there were no restoring force this motion would result in the free rotation of the hydroxyl group about the axis of the molecule. The absorption band between 16 and $26 \mu$ indicates, however, that the hydroxyl group is not entirely free, Fig. 6. At $26 \mu$ the absorption of the $\mathrm{KBr}$ foreprism made it impossible to work at longer wave-lengths although the band probably con-

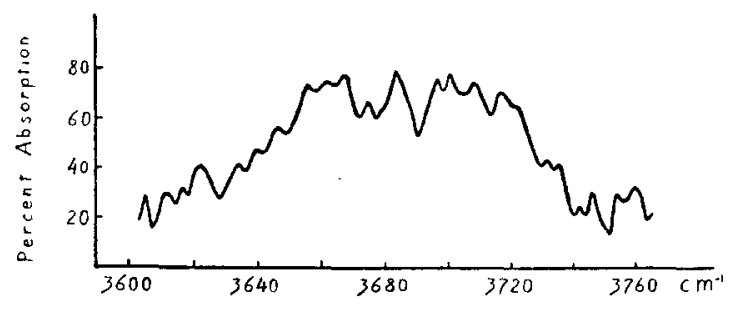

Fig. 5. The band $\nu_{7}$ at $3683 \mathrm{~cm}^{-1}$. 
tinues for some distance. There are a number of water lines in this region. These become more numerous and more intense as one proceeds toward longer wave-lengths. The curve is drawn with broken lines where the atmospheric absorption makes the readings uncertain.

Toward the high frequency edge of the band the spacing between the lines becomes greater and the lines fall off in intensity. Between 650 and $860 \mathrm{~cm}^{-1}$ is a group of very faint lines with a regular spacing of about $40 \mathrm{~cm}^{-1}$ which are probably a continuation of $\nu_{8}$, Fig. 7 . Although these lines could be observed with the $25 \mathrm{~cm}$ absorption cell, their structure was better defined when the meter cell was used. In the region between 700 and $630 \mathrm{~cm}^{-1}$ there is intense atmospheric absorption due to $\mathrm{CO}_{2}$. The line at $694.7 \mathrm{~cm}^{-1}$ was strong enough to stand out between the $\mathrm{CO}_{2}$ lines. It appeared to be fairly broad but it was impossible to give its exact structure. In addition to the fines shown in Fig. 7 , there is a region of absorption near 650 $\mathrm{cm}^{-1}$. This absorption, also, was broad but no part of it was strong enough to stand out between the $\mathrm{CO}_{2}$ lines. The frequencies of the most important lines of $\nu_{8}$ are listed in Table III.

\section{Discussion of Results}

The energy of an asymmetric molecule has the form: $:^{12}$

$$
\begin{aligned}
E= & \left(n+\frac{1}{2}\right) h \nu+\frac{h^{2}}{8 \pi^{2}} \\
& \times\left\{\left(J^{2}+J\right)\left(\frac{1}{2 A}+\frac{1}{2 B}\right)+\left(\frac{1}{C}-\frac{1}{2 A}-\frac{1}{2 B}\right) W\right\},
\end{aligned}
$$

where $W$ is a parameter which makes the energy matrix diagonal and is not in general an integer. When the values of $W$ are computed for the methyl alcohol molecule it turns out that only in the lowest states do they differ appreciably from squares of integers. This results in a displacement and splitting of the lowest levels but the effect is negligible for values of $W$ greater than 4 . This splitting affects the structure of the perpendicular bands slightly but, except for a slight broadening of the lines, it is unimportant in the parallel bands.

\footnotetext{
${ }^{12}$ H. H. Nielsen, Phys. Rev. 38, 1432 (1931).
}

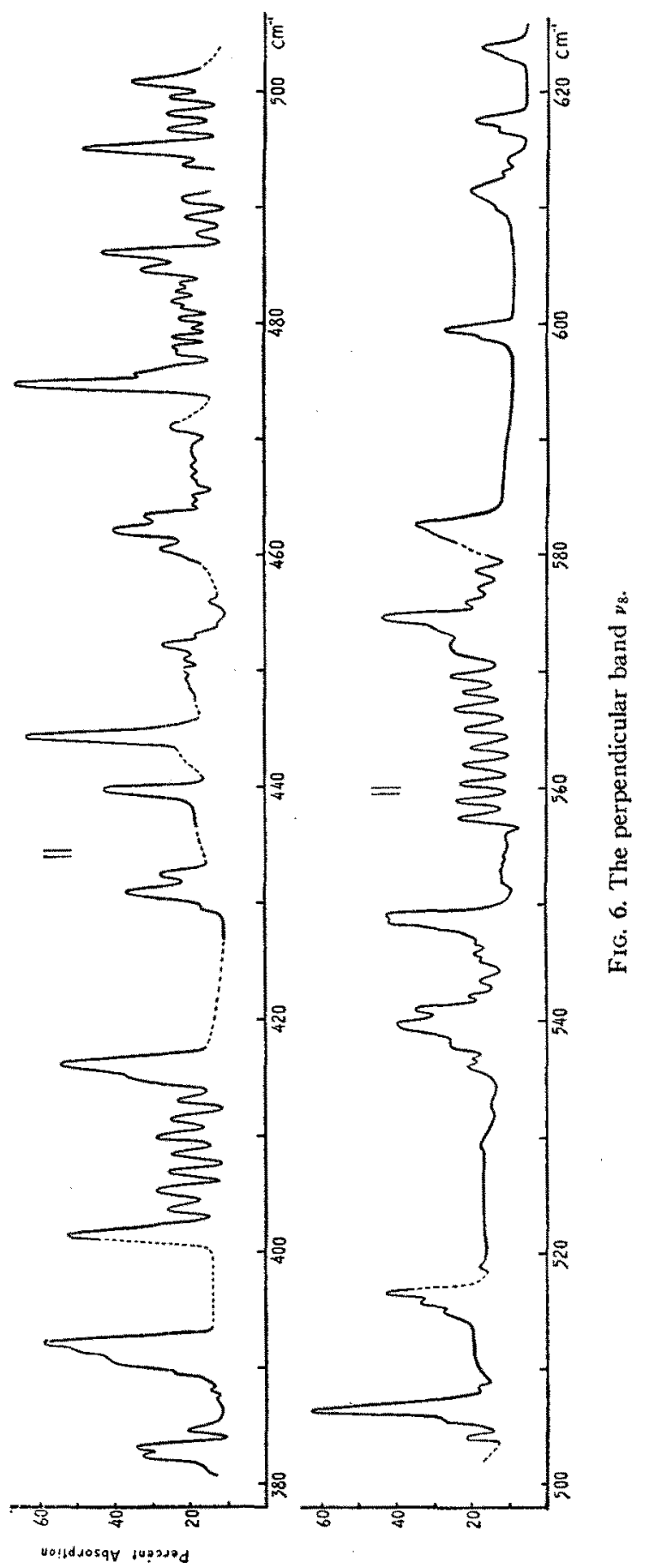

From the fine structure of the $1034.18 \mathrm{~cm}^{-1}$ parallel band an average value of the two largest moments of inertia of the molecule could be obtained. From further assumptions about the internuclear distances, using infra-red data on 
TABLE III. Lines of the perpendicular band $\nu_{8}$.

\begin{tabular}{lll}
\hline $382.45 \mathrm{~cm}^{-1}$ & $474.65 \mathrm{~cm}^{-1}$ & $582.60 \mathrm{~cm}^{-1}$ \\
383.20 & 484.60 & 599.50 \\
384.65 & 486.05 & 611.45 \\
392.15 & 494.95 & 617.55 \\
401.45 & 500.80 & 623.90 \\
416.10 & 506.40 & $(650)$ \\
439.70 & 515.73 & 694.7 \\
444.30 & 516.42 & 736.7 \\
452.20 & 539.60 & 778.3 \\
460.55 & 540.90 & 780.0 \\
462.20 & 548.65 & 820.6 \\
463.38 & 574.50 & 860.1 \\
\hline
\end{tabular}

the methyl halides ${ }^{13}$ and the water molecule, ${ }^{14}$ it was possible to compute $A$ and $B$ with a fair degree of accuracy. $A=35.18 \times 10^{-40} \mathrm{~g} \mathrm{~cm}^{2}$ and $B=33.83 \times 10^{-40} \mathrm{~g} \mathrm{~cm}^{2}$. The $\mathrm{C}-\mathrm{H}$ and $\mathrm{H}-\mathrm{H}$ distances in the methyl group were assumed to be $1.11 \mathrm{~A}$ and $1.81 \mathrm{~A}$, respectively. The $\mathrm{O}-\mathrm{H}$ distance was taken to be $0.9558 \mathrm{~A}$ as in water. In their experiments on electron diffraction Pauling and his co-workers ${ }^{15}$ give $1.44 \pm 0.02 \mathrm{~A}$ for the $\mathrm{C}-\mathrm{O}$ distance in $\mathrm{CH}_{3} \mathrm{ONH}_{2}$. Results from the moments of inertia of methyl alcohol indicate that the $\mathrm{C}-\mathrm{O}$ distance in methyl alcohol is between 1.42 and $1.43 \mathrm{~A}$ and the angle between the $\mathrm{O}-\mathrm{H}$ and the $\mathrm{C}-\mathrm{O}$ bonds lies between $105^{\circ}$ and $115^{\circ}$. These values cannot be known exactly until a more exact measurement of the least moment of inertia $C$ is made. Although the present observations do not yield $C$ directly its value can be estimated from the dimensions of the molecule. In making computations on the free rotation of the two parts of the molecule it is also necessary to know the approximate values of the moments of inertia of the hydroxyl and

${ }_{13}$ M. Johnston and D. M. Dennison, Phys. Rev. 48, 868 (1935).

${ }_{14}$ H. M. Randall, D. M. Dennison, N. Ginsburg, L. R. Weber, Phys. Rev, 52, 160 (1937).

${ }^{15}$ L. O. Brockway, J. Y. Beach, L. Pauling, Am. Chem. J. 57, 2693 (1935). methyl groups separately about the figure axis of the molecule. For the hydroxyl group $C_{1}$ is between 1.3 and $1.4 \times 10^{-40} \mathrm{~g} \mathrm{~cm}^{2}$; for the methyl group $C_{2}=5.4 \times 10^{-40} \mathrm{~g} \mathrm{~cm}^{2}$. The moment of inertia $C$ is equal to the sum of $C_{1}$ and $C_{2}$ and lies between 6.7 and $6.8 \mathrm{~g} \mathrm{~cm}^{2}$.

The perpendicular band $\nu_{8}$ is a single frequency and should be unaffected by the internal angular momentum which changes the spacing in the double perpendicular bands of symmetric molecules. A simple calculation shows that if the hydroxyl group were completely bound one should expect an ordinary perpendicular band having a spacing of approximately $6.5 \mathrm{~cm}^{-1}$. If, however, the hydroxyl group were a free rotator one should expect a series of rotation bands with a spacing of about $40 \mathrm{~cm}^{-1}$ between each band. The low frequency band shows an irregular spacing which increases from about $10 \mathrm{~cm}^{-1}$ to $30 \mathrm{~cm}^{-1}$ in the region from 380 to $600 \mathrm{~cm}^{-1}$ and finally has a spacing of about $40 \mathrm{~cm}^{-1}$ between 600 and $860 \mathrm{~cm}^{-1}$. The increased spacing toward higher frequencies probably indicates that for higher energies the hydroxyl group is approaching rotation levels.

\section{Free Rotation Bands of Methyl Alcohol}

The problem of the torsion oscillator-rotator has already been treated by Nielsen. ${ }^{16}$ For simplicity it will be assumed that the hydroxyl group can be approximated by a symmetrical top. Then the whole molecule can be represented by two symmetrical rotators having a common $C$ axis. It will be convenient to refer to the two systems with the subscripts 1 and 2 . The positions of the two parts of the molecule with respect to a set of fixed axes is best described by

${ }^{16}$ H. H. Nielsen, Phys. Rev. 40, 445 (1932).

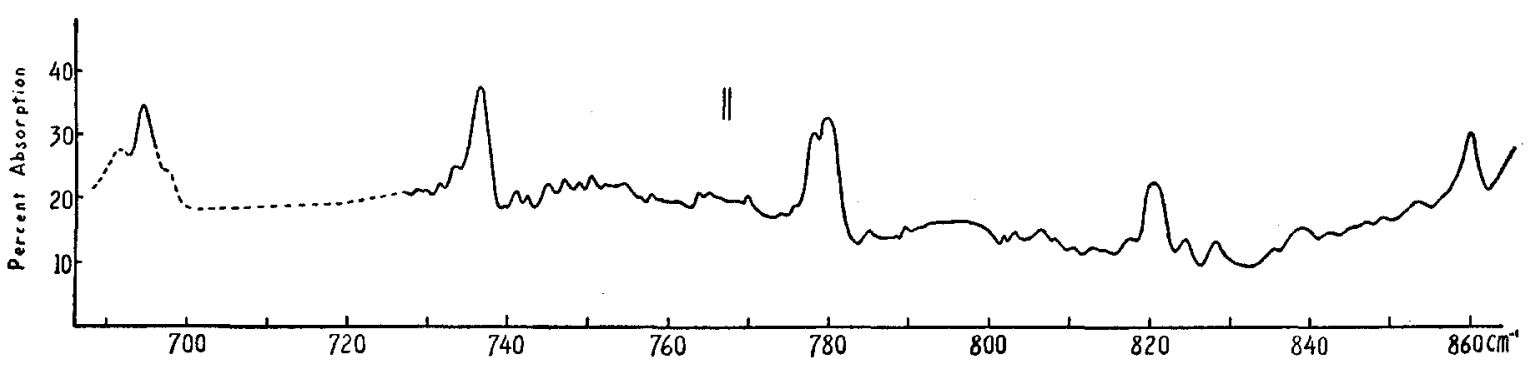

FIG. 7. Rotation lines of the band $\nu_{8}$. 
the Eulerean angles, $\theta, \psi, \varphi_{1}$ and $\theta, \psi, \varphi_{2}$; where $\theta$ is the angle between the fixed $Z$ axis and the $C$ axis of the molecule, $\psi$ is the angle which describes the precession of the molecule, $\varphi_{1}$ and $\varphi_{2}$ are the angles of rotation about the $C$ axis. Following Nielsen's procedure it will be advantageous to introduce the quantities $\varphi$ and $\alpha$ defined as follows : $\varphi=\left[\left(C_{1} \varphi_{1}+C_{2} \varphi_{2}\right) / C\right], \alpha=\varphi_{2}$ $-\varphi_{1}$, where $C_{1}$ and $C_{2}$ are the moments of inertia of the two parts of the molecule and $C_{1}+C_{2}=C$, the total moment of inertia about the figure axis of the molecule. Since the potential energy depends only upon the angular displacement between the two parts of the molecule it is a function of $\alpha$ only.

Proceeding as Nielsen did the Schrodinger equation is separable, one part giving the energy of rotation of the molecule as a whole:

$$
E_{1}=\frac{h^{2}}{8 \pi^{2}}\left\{\frac{J^{2}+J}{A}+\left(\frac{1}{C}-\frac{1}{A}\right) K^{2}\right\} .
$$

The other part leads to the differential equation:

$$
\frac{d^{2} M}{d \alpha^{2}}+\frac{8 \pi^{2} C_{1} C_{2}}{h^{2} C}\left\{E_{2}-V(\alpha)\right\} M=0 .
$$

Since the hydroxyl hydrogen atom has three positions of equilibrium between the hydrogen atoms of the methyl group the simplest potential function is: $V=V_{0}(1-\cos 3 \alpha)$. This leads to the Mathieu equation. As was pointed out by Nielsen exact solutions for this equation are known only for every third state. The intermediate solutions can only be estimated.

It is of interest to investigate the solutions for extreme values of the potential. If the barrier is indefinitely high there will be simple harmonic motion. This will give rise to an ordinary perpendicular band with a spacing $\left(h / 4 \pi^{2}\right)(1 / C-1 / A)$. If there is no barrier, however, there will be free rotation of the two parts of the molecule. Then the energy of the molecule may be written:

$$
E=\frac{h^{2}}{8 \pi^{2}}\left\{\frac{J^{2}+J-K^{2}}{A}+\frac{\lambda_{1}{ }^{2}}{C_{1}}+\frac{\lambda_{2}{ }^{2}}{C_{2}}\right\} .
$$

The eigenfunction has the form:

$$
\psi=F(\theta) e^{i N \psi} e^{i \lambda_{1} \varphi_{1}} e^{i \lambda_{2} \varphi_{2}},
$$

where $F(\theta)$ is the hypergeometric function. The notation used here is somewhat different from that employed by Nielsen. $\lambda_{1}$ and $\lambda_{2}$ are the azimuthal quantum numbers for the two parts of the top and may take on all integral values. $K$ is the total azimuthal quantum number and $|K|=\left|\lambda_{1}+\lambda_{2}\right|$.

The selection rules are similar to those obtained for ordinary perpendicular bands. If the electric moment changes in the system denoted by the subscript 1: $\Delta J=0, \pm 1, \Delta K= \pm 1, \Delta \lambda_{1}= \pm 1$, $\Delta \lambda_{2}=0$. If the electric moment changes in the other part of the molecule, then $\Delta \lambda_{1}=0$ and $\Delta \lambda_{2}= \pm 1$. In the following discussion it will be assumed that the electric moment changes in the hydroxyl group, which will be assigned the subscript 1.

Using these selection rules the zero branches on the low frequency side of the center of the band have the frequencies:

$$
\nu_{\lambda_{1} \lambda_{2} K}^{\lambda_{1}-1 \lambda_{2} K-1} J_{J}=\frac{h}{4 \pi^{2}}\left\{\frac{\lambda_{1}-\frac{1}{2}}{C_{1}}-\frac{K-\frac{1}{2}}{A}\right\}
$$

where

$$
\left|\lambda_{1}+\lambda_{2}\right|=|K| \text {. }
$$

The fine structure lines associated with the $K$ th zero branch have the frequencies:

High frequency side of the center

$$
\nu_{\lambda_{1} \lambda_{2} K}^{\lambda_{1}-1} \lambda_{2} K-1 J-1=\frac{h}{4 \pi^{2}}\left\{\frac{\lambda_{1}-\frac{1}{2}}{C_{1}}-\frac{K-\frac{1}{2}-J}{A}\right\}
$$

where

$$
|J| \geq|K| \text {. }
$$

Low frequency side of the center

$$
\nu_{\lambda_{1} \lambda_{2} K}^{\lambda_{1}-1 \lambda_{2} K-1 J} J_{-1}=\frac{h}{4 \pi^{2}}\left\{\frac{\lambda_{1}-\frac{1}{2}}{C_{1}}-\frac{K-\frac{1}{2}+J}{A}\right\}
$$

where

$$
|J-1| \geq|K| \text {. }
$$

Since $J$ is never less than $K$ certain of the fine structure lines will be missing. It is the same situation that arises in perpendicular bands of symmetric molecules and the band is constructed in the same way. On the low frequency side the first zero branch has the first rotation line on the low frequency side missing. The second zero branch has two lines missing on the low frequency side and one on the high frequency side. On the high frequency side the situation is just the reverse. A typical rotation band showing 
how the zero branches and fine structure lines go together is drawn in Fig. 8. The asymmetry of the band will be explained later.

It is apparent from these equations and the diagram that the fine structure lines and the zero branches have the same spacing and in most cases a number of lines coincide. In computing the intensities it is necessary to find the sum of the intensities of all the lines falling at a particular place. It will be convenient to find the intensities of the zero branches and fine structure lines separately. The intensity formulae for perpendicular bands given by Dennison ${ }^{6}$ may be adapted for use here. For the zero branches on the low frequency side of the center the intensity. formula is :

$$
\begin{aligned}
& I_{\lambda_{1} \lambda_{2} K}^{\lambda_{1}-1 \lambda_{2} K-1 J}=A[x] \sum_{J=K}^{\infty} \frac{(2 J+1)(J+K)(J-K+1)}{2 J(J+1)} \\
& \times \exp \left(-\sigma\left[J^{2}+J-(K-1)^{2}\right.\right. \\
& \left.\left.+\gamma_{1}\left(\lambda_{1}-1\right)^{2}+\gamma_{2} \lambda_{2}^{2}\right]\right), \\
& \text { where } \quad \sigma=\frac{h^{2}}{8 \pi^{2} A k T}, \quad \gamma_{1}=\frac{A}{C_{1}}, \quad \gamma_{2}=\frac{A}{C_{2}} \text {. }
\end{aligned}
$$

$A$ is a constant which depends upon the popula-

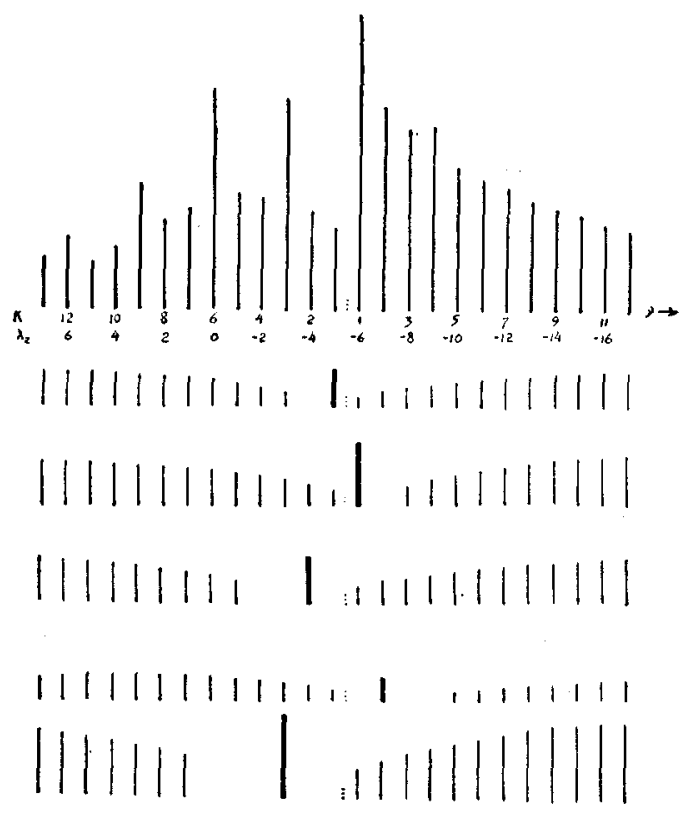

11111111111111111111

FIG. 8. The free rotation band $\lambda_{1}=6$. tion in the initial state and the Boltzmann factor. The quantity $[x]$ is equal to one except when the quantum number $\lambda_{2}$ is zero or any multiple of three, in which case $[x]$ takes on the value two. This enhancement of every third line is due to the presence of the three protons (like particles with spin $\left.\frac{1}{2}(h / 2 \pi)\right)$ in the methyl group which doubles the population in every third state.

In the approximation needed here it is convenient to change the sum into an integral:

$$
\begin{aligned}
& I_{\lambda_{1} \lambda_{2} K}^{\lambda_{1}-1 \lambda_{2} K-1 J}=\frac{A}{2}[x] \\
& \times \exp \left(-\sigma\left[\gamma_{1}\left(\lambda_{1}-1\right)^{2}+\gamma_{2} \lambda_{2}{ }^{2}-(K-1)^{2}\right]\right) \\
& \times\left\{\frac{e^{-\sigma\left(K^{2}+K\right)}}{\sigma}-\left(K^{2}-K\right) \int_{\sigma\left(K^{2}+K\right)}^{\infty} \frac{e^{-u}}{u} d u\right\} .
\end{aligned}
$$

The integral which occurs here is the logarithmic integral for which tables have been computed. A similar expression is obtained for the zero branches on the high frequency side:

$$
\begin{aligned}
& \underset{\lambda_{1}}{\lambda_{1}-1} \lambda_{2} K \quad J \quad J \quad J=\frac{A}{2}[x] \\
& \times \exp \left(-\sigma\left[\gamma_{1}\left(\lambda_{1}-1\right)^{2}+\gamma_{2} \lambda_{2}{ }^{2}-K^{2}\right]\right) \\
& \times\left\{\frac{e^{-\sigma\left(K^{2}-K\right)}}{\sigma}-\left(K^{2}-K\right) \int_{\sigma\left(K^{2}-K\right)}^{\infty} \frac{e^{-u}}{u} d u\right\} .
\end{aligned}
$$

The intensities of the fine structure lines can. best be treated in two parts. First consider the fine structure associated with zero branches on the opposite side of the center of the band, and then consider the fine structure associated with zero branches on the same side of the center. In either case it is necessary to sum over all rotation lines of that class falling on the $K$ th zero branch.

Consider all rotation lines on the high frequency side of the band having zero branches on the low frequency side, which fall on the $K$ th zero branch on the high frequency side. The $K$ th zero branch has the frequency:

$$
\nu_{\lambda_{1} \lambda_{2} K-1 J}^{\lambda_{1}-1 \lambda_{2} K} \quad J=\frac{h}{4 \pi^{2}}\left\{\frac{\lambda_{1}-\frac{1}{2}}{C_{1}}+\frac{K-\frac{1}{2}}{A}\right\},
$$

where $K-1=-\left(\lambda_{1}+\lambda_{2}\right)$. The fine structure line 
associated with the $K^{\prime}$ th zero branch on the low frequency side has the frequency:

$$
\underset{\lambda_{1} \lambda_{2^{\prime}} K^{\prime}}{\lambda_{\lambda_{1}-1} \lambda_{\lambda^{\prime}} K^{\prime}-1 J-1}=\frac{h}{4 \pi^{2}}\left\{\frac{\lambda_{1}-\frac{1}{2}}{C_{1}}-\frac{K^{\prime}-\frac{1}{2}-J}{A}\right\},
$$

where $K^{\prime}=\lambda_{1}+\lambda_{2}{ }^{\prime}$ and $|J| \geq\left|K^{\prime}\right|$. It is clear that this fine structure line coincides with the $K$ th zero branch if $K^{\prime}=J-K+1$ and $\lambda_{2}{ }^{\prime}=J-K$ $+1-\lambda_{1}$. Each fine structure line has the intensity:

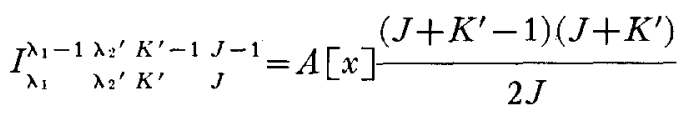

$$
\begin{aligned}
& \times \exp \left(-\sigma\left[J^{2}-J-\left(K^{\prime}-1\right)^{2}\right.\right. \\
& \left.\left.+\gamma_{1}\left(\lambda_{1}-1\right)^{2}+\gamma_{2} \lambda_{2}{ }^{\prime 2}\right]\right) \\
& =A[x] e^{-\sigma b} \frac{(2 J-K)(2 J-K+1)}{2 J} \\
& \times \exp \left(-\sigma \gamma_{2}(J+a)^{2}\right) \text {, }
\end{aligned}
$$

where

$$
\begin{aligned}
& a=\frac{2 K-1-2 \gamma_{2}\left(K+\lambda_{1}-1\right)}{2 \gamma_{2}}, \\
& b=\gamma_{1}\left(\lambda_{1}-1\right)^{2}+\gamma_{2}\left[\left(K+\lambda_{1}-1\right)^{2}-a^{2}\right]-K^{2} .
\end{aligned}
$$

The intensity of all the fine structure lines falling on the $K$ th zero branch may be found by summing this expression over $J$, from $J=K$ to infinity. It will be more convenient, however, to use the integral form, where $4 / 3$ has been written for the average value of $[x]$.

$$
\begin{aligned}
I=\frac{4}{3} A e^{-\sigma b} \int_{K}^{\infty}[2(J+a)- & \left.(2 K-1+2 a)+\frac{K^{2}-K}{2 J}\right] \\
& \times \exp \left(-\sigma \gamma_{2}(J+a)^{2}\right) d J .
\end{aligned}
$$

The first term may be integrated at once and the other two may be written in a simpler form:

$$
\begin{aligned}
I=-A e^{-\sigma b} & \left\{\frac{e^{-\sigma \gamma_{3}(K+a)^{2}}}{\sigma \gamma_{2}}\right. \\
& -\frac{2 K-1+2 a}{\left(\sigma \gamma_{2}\right)^{\frac{2}{2}}} \int_{(\sigma \gamma 2)^{\frac{1}{2}}(K+a)}^{\infty} e^{-x^{2} d x} \\
& \left.\quad+\frac{K^{2}-K}{2} \int_{(\sigma \gamma 2)^{\frac{1}{2}}(K+a)}^{\infty} \frac{e^{-x^{2}}}{x+\left(\sigma \gamma_{2}\right)^{\frac{1}{2}} a} d x\right\}
\end{aligned}
$$

The second integral is the probability integral for which tables have been computed. The third may be evaluated numerically.

Exactly the same expression may be obtained for the intensities of the fine structure lines on the low frequency side which are associated with zero branches on the high frequency side. Here, however,

$$
a=\frac{2 K-1-2 \gamma_{2}\left(K-\lambda_{1}\right)}{2 \gamma_{2}}
$$

and

$$
b=\gamma_{1}\left(\lambda_{1}-1\right)^{2}+\gamma_{2}\left[\left(K-\lambda_{1}\right)^{2}-a^{2}\right]-(K-1)^{2} .
$$

\begin{tabular}{|c|c|c|c|c|c|c|c|}
\hline \multicolumn{4}{|c|}{ 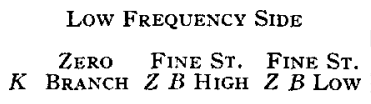 } & \multicolumn{4}{|c|}{ 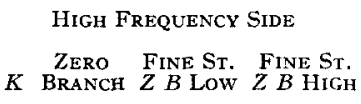 } \\
\hline $\begin{array}{r}1 \\
2 \\
3 \\
4 \\
5 \\
6 \\
7 \\
8 \\
9 \\
10 \\
11 \\
12 \\
13 \\
14 \\
15 \\
16 \\
17 \\
18\end{array}$ & $\begin{array}{r}7.78 \\
9.85 \\
21.30 \\
11.50 \\
11.70 \\
22.50 \\
10.40 \\
8.98 \\
14.98 \\
5.90 \\
4.52 \\
6.52 \\
2.16 \\
1.49 \\
1.85 \\
0.54\end{array}$ & 0.58 & $\begin{array}{l}0.15 \\
0.34 \\
0.42 \\
0.94 \\
1.01 \\
1.04 \\
0.86\end{array}$ & $\begin{array}{r}1 \\
2 \\
3 \\
4 \\
5 \\
6 \\
7 \\
.8 \\
9 \\
10 \\
11 \\
12 \\
13 \\
14\end{array}$ & $\begin{array}{r}12.60 \\
4.50 \\
3.03 \\
3.89 \\
1.20\end{array}$ & $\begin{array}{r}17.21 \\
16.18 \\
15.23 \\
14.41 \\
13.45 \\
12.82 \\
11.37 \\
9.82 \\
8.44 \\
6.66\end{array}$ & $\overline{-}$ \\
\hline
\end{tabular}

In some cases $a$ is negative and greater than $K$. Here the integral does not well approximate the sum. It is then more convenient to sum the first few terms until $J \geq a$. The integral form may be used for the remaining terms.

Now consider all the fine structure lines on the low frequency side associated with zero branches also on the low frequency side. The intensity of one of these lines which is associated with the $K^{\prime}$ th zero branch is :

$$
\begin{array}{r}
I_{\lambda_{1}}^{\lambda_{1}-1 \lambda_{2} \lambda_{2^{\prime}}, K^{\prime}-1} \underset{J-1}{\lambda^{\prime}}=A[x] \frac{\left(J-K^{\prime}+1\right)\left(J-K^{\prime}\right)}{2 J} \\
\times \exp \left(-\sigma\left[J^{2}+J-\left(K^{\prime}-1\right)^{2}\right.\right. \\
\left.\left.+\gamma_{1}\left(\lambda_{1}-1\right)^{2}+\gamma_{2} \lambda_{2}{ }^{2}\right]\right)
\end{array}
$$

A fine structure line associated with the $K^{\prime}$ th TABLE IV. Intensities of lines in the rotation band $\lambda_{1}=6$. 
zero branch will coincide with the $K$ th zero branch if $K^{\prime}=K-J$ and $\lambda_{2}{ }^{\prime}=K-J-\lambda_{1}$. It is only high $K$ values, Fig. 8 , which receive contributions from this class of fine structure since $J \geq K^{\prime}+1$, or $J \geq(K+1) / 2$ if $K$ is odd and $J \geq(K+2) / 2$ if $K$ is even. Also, since $J=K-K^{\prime}$, only those values of $J \leq K-1$ can give contributions to the $K$ th zero branch. The intensity of all these fine structure lines falling on the $K$ th zero branch is:

$$
I=A e^{-\sigma b} \sum_{\substack{J=(K+1) / 2 \\ J=(K+2) / 2}}^{K-1} \frac{(2 J-K)(2 J-K+1)}{2 J} \text { even. }
$$

$$
\text { where } \quad a=\frac{2 K-1-2 \gamma_{2}\left(K-\lambda_{1}\right)}{2 \gamma_{2}}
$$

and

$$
b=\gamma_{1}\left(\lambda_{1}-1\right)^{2}+\gamma_{2}\left[\left(K-\lambda_{1}\right)^{2}-a^{2}\right]-(K-1)^{2} .
$$

The same sum is obtained for the intensities of the fine structure lines on the high frequency side of the band which are associated with zero branches also on the high frequency side. In this case, however,

$$
a=\frac{2 K-1-2 \gamma_{2}\left(K+\lambda_{1}-1\right)}{2 \gamma_{2}}
$$

and $\quad b=\gamma_{1}\left(\lambda_{1}-1\right)^{2}+\gamma_{2}\left[\left(K+\lambda_{1}-1\right)^{2}-a^{2}\right]-K^{2}$.

Since it is only in states of high excitation that the hydroxyl group approaches free rotation the intensities of some of the lines in the band where $\lambda_{1}=6$ are computed in Table IV. This band is drawn in Fig. 8. The quantum number $K$ changes from $K-1$ to $K$ for the lines on the low frequency side of the center of the band and from $K$ to $K-1$ for the lines on the high frequency side. In each rotation band there are two particularly strong zero branch lines having the quantum number $\lambda_{2}=0$ and -3 . In the first rotation band where $\lambda_{1}=1$ these strong lines would occur at the center of the band and the fine structure lines would be distributed evenly on both sides. In each successive band, however, these strong lines are displaced farther from the center toward lower frequencies by an amount $h /\left(4 \pi^{2} A\right)$. In high rotation states this displacement brings most of the important zero branches on the low frequency side of the center. Thus the important contributions from the fine structure in the high rotation bands is that associated with zero branches on the low frequency side. These fine structure lines always begin at a distance $h /\left(8 \pi^{2} A\right)$ from the center of the band on the high frequency side but they build up more slowly on the low frequency side. For this reason the bands have an unsymmetrical appearance and are very intense on the high frequency side since so many fine structure lines coincide here. This effect is enhanced in each successive band as $\lambda_{1}$ increases.

These bands do not compare very well with the structure of the lines in Fig. 7. These are not single lines, however. It may be that, when interactions between the two parts of the molecule are considered, the rotation bands will have quite a different form.

The results of the present investigation are highly suggestive from a qualitative point of view. It has been shown that, if the motion of the hydroxyl group were entirely free, one should expect a series of rotation bands with a separation of about $40 \mathrm{~cm}^{-1}$ between each band. This is clearly not the case here. The very fact of the existence of a low frequency band shows that the levels must in some measure possess a vibrationlike character. This indicates that the hydroxyl atom is confined within a threefold potential barrier produced by the three hydrogen atoms of the methyl group. If this barrier were very high the particle would vibrate in one of its positions of equilibrium and give rise to a typical perpendicular band. This band would not be affected by vibration-rotation interaction and should have a spacing of about $6.5 \mathrm{~cm}^{-1}$. The spacing in the low frequency band is between these two extremes. At the low frequency edge of the band the spacing approaches the bound case but near the high frequency edge the distance between the lines indicates that the hydroxyl atom is nearly free.

While the evidence is not at all conclusive, it suggests that the height of the barrier might be estimated by correlating it with that part of the band where the distance between the lines begins to approach the spacing expected on the basis 
of free rotation. This would appear to correspond to a quantum number of the order of magnitude of 5 in the energy of the free hydroxyl rotator. This estimate of the barrier is accordingly between 400 and $600 \mathrm{~cm}^{-1}$. A study of the far infra-red would be helpful in fixing this value.

It is beyond the scope of the present paper to discuss the transition between vibrational and rotational states. Since the potential function of the alcohol molecule is intermediate between the two extremes discussed here, it is clear that both the rotational and vibrational states are complicated by the presence of the three minimum potential barrier.

In conclusion the authors wish to express their gratitude to Professor D. M. Dennison for his interest in the work and for many helpful discussions during the development of the theory.

\title{
The Infra-Red Absorption Spectra of $\mathrm{CH}_{3} \mathrm{OD}$ and $\mathrm{CH}_{2} \mathrm{DOD}$
}

\author{
E. F. Barker and G. Bosschieter \\ Department of Physics, University of Michigan, Ann Arbor, Michigan
}

(Received June 23, 1938)

\begin{abstract}
The infra-red absorption spectra of $\mathrm{CH}_{3} \mathrm{OD}$ and $\mathrm{CH}_{2} \mathrm{DOD}$ between $2.5 \mu$ and $24 \mu$ have been examined with a $\mathrm{KBr}$ prism spectrometer, and with appropriate gratings. The observed bands represent all of the fundamental vibrations except the one of lowest frequency which is associated with torsional vibrations about the $\mathrm{C}-\mathrm{O}$ bond. Since these molecules depart only slightly from axial symmetry, the bands, with the exception of three due to the hydroxyl radical, correspond in position and appearance to those of the methyl halides. The rotational structure for the $10 \mu$ band $\left(\nu_{5}\right)$ of $\mathrm{CH}_{3} \mathrm{OD}$ has been resolved, and partial resolution is obtained in some other bands. The band $\nu_{5}$ in
\end{abstract}

$T$ HE Raman spectra of ordinary methyl alcohol in the liquid phase, and of two methyl alcohols containing deuterium, have recently been described by Halford, Anderson and Kissin. ${ }^{1}$ While the particular modes of vibration to be associated with each observed frequency may be decided almost by inspection, it is only through measurements in the infra-red that the character of the motions is revealed. Moreover the true molecular frequencies must be observed for the gaseous state. A comparison of infra-red data for the gases with Raman data for the liquids yields information of interest particularly in connection with the problem of association.

The molecule of methyl alcohol departs slightly from axial symmetry, since the line

\footnotetext{
${ }^{1}$ Halford, Anderson and Kissin, J. Chem. Phys. 5, 927 (1937). See also Mizushima, Morino and Okamoto, Bull. Chem. Soc. Japan 11, 698 (1936).
}

$\mathrm{CH}_{2}$ DOD has two components arising probably from two forms of the molecule in which the hydroxyl $\mathrm{D}$ atom occupies different valleys of the threefold potential. The deformation vibration $\left(\nu_{7}\right)$ is single for $\mathrm{CH}_{3} \mathrm{OD}$ but has four components in $\mathrm{CH}_{2} \mathrm{DOD}$, indicating a separation of levels which for the former molecule are degenerate. A comparison of the frequencies obtained with gaseous and liquid samples indicates large displacements of the bands arising from the hydroxyl valence and deformation vibrations, the former toward greater wave-lengths in the liquid, and the latter toward smaller wave-lengths.

connecting the atoms $\mathrm{O}$ and $\mathrm{H}$ is nearly perpendicular to that connecting $\mathrm{C}$ and $\mathrm{O}$. The minimum moment of inertia corresponds to an axis which is very nearly parallel to the $\mathrm{C}-\mathrm{O}$ line, and which, for convenience, may be referred to as the symmetry axis. The relatively large electric moment may be attributed principally to the hydroxyl group. Twelve fundamental vibrations are to be expected, some of which occur in pairs, corresponding to two dimensional oscillators with nearly equal frequencies in the plane determined by the atoms $\mathrm{C}, \mathrm{O}$ and $\mathrm{H}$, and normal to it. Six of these are associated primarily with the methyl radical, three with the $\mathrm{C}-\mathrm{O}$ bond, and three with the hydroxyl group, the first nine being conveniently designated as in the methyl halides. ${ }^{2}$

\footnotetext{
${ }^{2}$ Dennison, Rev. Mod. Phys. 3, 280 (1931).
} 